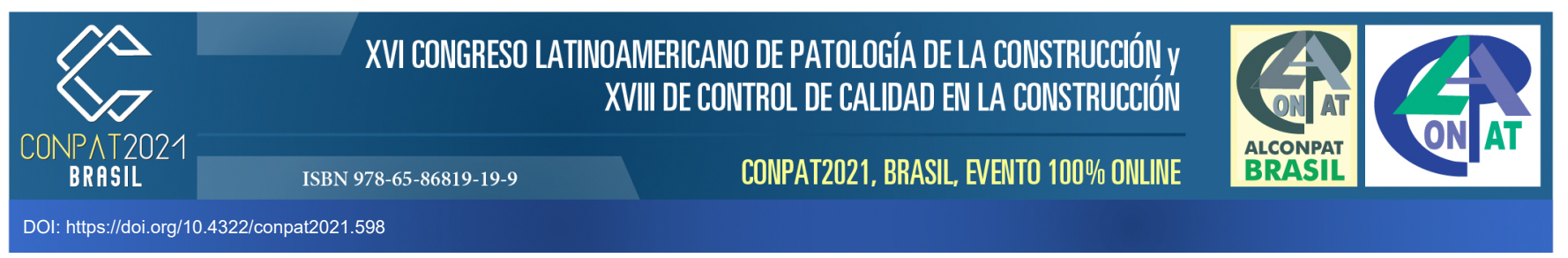

\title{
EVALUACIÓN DEL EFECTO DE NANOPARTÍCULAS BASE SILICIO EN MORTEROS SOMETIDOS AL ATAQUE FÍSICO DE SULFATO DE SODIO
}

\author{
D. L. Silva ${ }^{1,2^{*}}$; G. Fajardo-San Miguel ${ }^{1}$; G. Escadeillas ${ }^{2}$, D. Cruz-Moreno ${ }^{1}$. \\ *Autor de Contacto: gerardo.fajardosn@uanl.edu.mx \\ ${ }^{1}$ Universidad Autónoma de Nuevo León. Facultad de Ingeniería Civil, San Nicolás de los Garza, México. \\ ${ }^{2}$ LMDC, Université de Toulouse, INSA, UPS Génie Civil, Toulouse, France.
}

\begin{abstract}
RESUMEN
El presente estudio consiste en evaluar la factibilidad de tratamientos superficiales con nanopartículas de silicio simple (NS) y funcionalizada (NF) en morteros de cemento para mitigar físico de sulfatos. Se sometieron morteros de cemento portland ordinario (CPO) y cemento resistente a los sulfatos (RS) a tres tipos de tratamientos superficiales: T1 - con una solución al $0.1 \%$ de NS, aplicada mediante inmersión, en las primeras $72 \mathrm{~h}$ de hidratación, $\mathrm{T} 2$ - con una solución al $0.1 \%$ de NF, aplicada mediante cepillado a los 28 días de hidratación y T3 combinación del T1 con el T2. Posteriormente, los especímenes fueron parcialmente inmersos en una solución al 5\% de sulfato de sodio y sometidos a ciclos de mojado y secado. Además, se midió el ángulo de contacto superficial de los especímenes, la pérdida de masa y se realizó el análisis de sortividad. Finalmente, los resultados han demostrado que el tratamiento superficial T3 presenta un mejor comportamiento ante al ataque físico de sulfatos. Posiblemente, lo anterior se debe a que hasta el segundo mes de exposición, los especímenes del tratamiento T3, presentaron una menor absorción, como se observó en los análisis de pérdida de masa y sortividad, impidiendo el ingreso de sulfato hacia el interior de la matriz y postergando su migración hacia la zona de evaporación.
\end{abstract}

Palabras-clave: Nanopartículas base silicio; Ataque físico de sulfatos; Durabilidad.

ABSTRACT 
The present study consists of evaluating the feasibility of surface treatments with simple (NS) and functionalized (NF) silica nanoparticles in cement mortars to mitigate physical sulfate attack. Ordinary portland cement (CPO) and sulfate resistant cement (RS) mortars were subjected to three types of surface treatments: T1 - with a $0.1 \%$ solution of NS, applied by immersion, in the first $72 \mathrm{~h}$ of hydration, T2 - with a $0.1 \%$ solution of NF, applied by brushing at 28 days of hydration and T3 - combination of T1 with T2. Subsequently, the specimens were partially immersed in a $5 \%$ sodium sulfate solution and subjected to wetting and drying cycles. In addition, the surface contact angle of the specimens was measured, the mass loss and the sorptivity analysis was performed. Finally, the results have shown that the T3 surface treatment has a better behavior against the physical attack of sulfates. Possibly, this is due to the fact that until the second month of exposure, the T3 treatment specimens presented lower absorption, as observed in the analysis of loss of mass and sorptivity, preventing the entry of sulfate into the interior of the matrix and postponing its migration to the evaporation zone.

Keywords: silicon-based nanoparticles; Physical attack of sulfates; Durability.

\section{RESUMO}

O presente estudo consiste em avaliar a viabilidade de tratamentos de superfície com nanopartículas de silice simples (NS) e funcionalizadas (NF) em argamassas de cimento para mitigar ataque físico do sulfato. As argamassas de cimento portland comum (CPO) e de cimento resistente ao sulfato (RS) foram submetidas a três tipos de tratamentos de superfície, tais como: T1 - com solução $0,1 \%$ de NS, aplicada por imersão, nas primeiras $72 \mathrm{~h}$ de hidratação, T2 - com solução de NF 0,1\%, aplicada por escovação aos 28 dias de hidratação e T3 - combinação de T1 com T2. Posteriormente, os corpos-de-prova foram parcialmente imersos em solução de sulfato de sódio a 5\% e submetidos aos ciclos úmido e seco. Além disso, o ângulo de contato da superfície dos espécimes, a perda de massa e a análise de ordenação foram realizadas. Por fim, os resultados mostraram que o tratamento de superfície T3 apresenta um melhor comportamento contra o ataque físico de sulfatos. Possivelmente, isso se deve ao fato de que até o segundo mês de exposição, os corpos-de-prova do tratamento T3 apresentaram menor absorção, conforme observado na análise de perda de massa e ordenação, impedindo a entrada do sulfato no interior da matriz e postergando sua migração para a zona de evaporação.

Palavras-chave: Nanopartículas base silício; Ataque físico de sulfatos; Durabilidade.

\section{INTRODUCCIÓN}

Desde hace algunas décadas se creía que el ataque químico de sulfatos era el principal mecanismo de degradación de estructuras expuestas a los iones sulfatos. Sin embargo, el daño del ataque físico de sulfatos, debido a la cristalización de la sal en los poros de la estructura empezó a ser considerado por muchos investigadores (Zeidan et al., 2016; Nehdi et al., 2014). Este problema está asociado con la formación de incrustaciones en la superficie, debido a la absorción de soluciones salinas que se elevan por capilaridad a las superficies de evaporación de la estructura, asociado a ciclos repetidos de variación de humedad relativa y de temperatura. En particular, esto ocurre cuando la 
tasa de evaporación de la superficie del concreto excede la absorción de la solución por capilaridad (Haynes et al., 2011; Zeidan et al., 2016).

Cabe mencionar que, al contrario del ataque químico de sulfatos, en el ataque físico no se produce reacciones con los compuestos hidratados del cemento portland. En este caso, el sulfato de sodio, que es el más dañino al concreto, debido a ciclos de humedecimiento y secado produce un cambio de fase de la thenardita $\left(\mathrm{Na}_{2} \mathrm{SO}_{4}\right)$, que es la fase anhidra del sulfato de sodio, para la mirabilita $\left(\mathrm{Na}_{2} \mathrm{SO}_{4} \cdot 10 \mathrm{H}_{2} \mathrm{O}\right)$, que es la fase hidratada (Haynes et al., 2008). Fundamentalmente, este cambio de fase proporciona un aumento en el volumen de aproximadamente 314\% (Scherer, 2004, Thaulow y Sahu, 2004). El ataque físico de sulfatos suele pasar, principalmente, en estructuras marinas, debido a la evaporación del agua del mar y/o la ascensión capilar. Ribeiro et al. (2014) señalaron que el aumento de volumen debido a la cristalización de las sales aumenta las tensiones internas, resultando en pérdida de adherencia y cohesión de la pasta, agrietamiento y delaminación del concreto. Estos daños se limitan a la superficie de la matriz cementante que se encuentra en la zona de evaporación de la estructura, mientras que la porción que está inmersa se muestra totalmente sana (Yoshida, 2010). En contraste, Nehdi et al. (2014) reportaron que en la porción inmersa puede ocurrir el ataque químico de sulfato, favoreciendo el ataque doble de sulfatos en la estructura.

Con la nanotecnología, en lo cual es posible comprender y controlar la materia a nanoescala, a escala atómica y molecular, el uso de nanomateriales ha sido ampliamente estudiado en materiales cementantes durante los últimos años, para incrementar su durabilidad (Atahan y Dikme, 2011; Jalal et al., 2012; Li et al., 2017). El nanomaterial más empleado en materiales cementantes ha sido las nanopartículas base sílice - NS, en su forma simple y/o funcionalizada (NF). En su forma simple, cuando son añadidas a la matriz aún en el momento del mezclado, genera sitios de nucleación para la formación productos hidratados, promueve la reacción puzolánica con la portlandita, generando C-S-H secundario, y consecuentemente disminuyendo la permeabilidad (Tobón et al., 2015; Gómez-Zamorano y Castillo-Linton, 2016).

Por otro lado, cuando las NS son añadidas mediante tratamiento superficial, además del aporte mencionado anteriormente, ejercen el efecto fíller, conllevando al bloqueo de poros interconectados $\mathrm{y}$, por consiguiente, a la reducción de la permeabilidad, disminuyendo la penetración de agentes agresivos. En su forma funcionalizada, que se obtiene mediante modificación superficial de las NS, las NF son especialmente interesantes en tratamiento de superficies porque pueden actuar desarrollando superficies hidrofóbicas, autolimpiantes, bactericidas, etc., debido al anclaje que desarrollan ante la superficie (Cruz-Moreno et al., 2020). El presente trabajo busca determinar la factibilidad de aplicación del tratamiento superficial con NS y NF en la etapa de curado de morteros cemento portland ordinario - CPO y cemento resistente a los sulfatos - RS, como una técnica de prevención al ataque físico de sulfatos en morteros.

\section{PROCEDIMIENTO EXPERIMENTAL}

\subsection{Obtención de NS y NF}

La obtención de las NS y NF en laboratorio se llevó a cabo mediante la ruta sol-gel como ha sido reportado previamente (Fajardo et al., 2015; Cruz-Moreno et al., 2020). Este método es ampliamente utilizado en la producción de materiales nanométricos, por ser fácil de controlar y realizar.

\subsection{Caracterización de las NS y NF}


La caracterización de las NS y NF se llevó a cabo mediante las técnicas que se mencionan a continuación:

Espectrometría de fluorescencia de rayos X (FRX): con el fin de comprobar el grado de pureza de las NS y NF se realizó el análisis de espectrometría de fluorescencia de rayos X (FRX), que se llevó a cabo en un equipo PANanalytical Epsilon3-XL, en atmósfera inerte de nitrógeno. Para este análisis se utilizaron muestras en polvo, previamente secas a $110^{\circ} \mathrm{C}$.

Difracción de rayos $\mathrm{X}(\mathrm{DRX})$ : para identificar los compuestos presentes y la naturaleza del material se realizó el análisis de difracción de rayos $\mathrm{X}$, mediante el difractómetro PANallytical EMPYREAN con radiación $\mathrm{CuK} \alpha$, en atmósfera inerte de nitrógeno. El análisis se llevó a cabo en el rango de $2 \theta=10$ a $60^{\circ}$. Para este análisis se utilizaron muestras en polvo, previamente secas a $110^{\circ} \mathrm{C}$.

Espectroscopia de infrarrojo con transformada de Fourier (FTIR): para identificar los grupos funcionales de las NS y NF y subproductos de la síntesis, se llevó a cabo el análisis de espectroscopia de infrarrojo con transformada de Fourier (FTIR), en un equipo Thermo SCIENTIFIC NICOLET iS50 FTIR. Para este análisis, se preparó una dispersión en agua destilada al $0.1 \%$ de NS y NF. El análisis se realizó en el intervalo de longitud de onda de 500 a $4000 \mathrm{~cm}^{-1}$.

Microscopía electrónica de transmisión (TEM): para identificar la morfología y el tipo de las nanopartículas, se llevó a cabo el análisis de microscopía electrónica de transmisión (TEM), mediante el equipo Hitachi 7700. Para el análisis, se utilizaron muestras dispersas en alcohol etílico.

\subsection{Preparo y colado de especímenes}

Para la preparación de los morteros se utilizó como aglutinante el cemento portland ordinario CPO (que cumple con la especificación de la norma NMX-C-414-ONNCCE-2004, y es equivalente al tipo I de la norma ASTM C150 - 2007) y el cemento RS (que cumple con la especificación de la norma NMX-C-414-ONNCCE - 2004, y es equivalente al tipo V de la norma ASTM C150 2007). La Tabla 1 presenta la composición química del CPO y RS que fue obtenida mediante la espectrometría de fluorescencia de rayos $\mathrm{X}(\mathrm{FRX})$ en un equipo de PANanalytical modelo Epsilon3-XL, en atmósfera inerte de nitrógeno. La densidad del cemento se obtuvo mediante el multipicnómetro modelo QUANTACHROMEy la distribución de tamaño de partícula (DTP) fue obtenida mediante la técnica de difracción de rayo láser, con el analizador de partícula tri-láser MICROTRAC modelo S3500 (véase Tabla 1).

Tabla 1 - Composición química del CPO y RS

\begin{tabular}{|l|c|c|c|c|c|c|c|c|c|c|}
\hline $\begin{array}{c}\text { Composición } \\
(\%)\end{array}$ & $\mathbf{C a O}$ & $\mathbf{S i O}_{2}$ & $\mathbf{S O}_{3}$ & $\mathbf{A l}_{2} \mathbf{O}_{3}$ & $\mathbf{F e}_{2} \mathbf{O}_{3}$ & $\mathbf{K}_{2} \mathbf{O}$ & $\mathbf{M g O}$ & $\mathbf{T i O}_{2}$ & $\boldsymbol{\rho}\left(\mathbf{g} / \mathbf{c m}^{3}\right)$ & $\mathbf{D T P}(\mu \mathrm{m})$ \\
\hline CPO 40 & 50.642 & 36.67 & 5.049 & 3.320 & 2.056 & 0,971 & 0.682 & 0.206 & 3.0 & 19.47 \\
\hline RS & 50.140 & 38.085 & 4.779 & 2.567 & 2.728 & 0.591 & 0.678 & 0.144 & 3.1 & 19.25 \\
\hline
\end{tabular}

Como agregado fino_se utilizó una arena estándar comercial (sílice de Ottawa), que según la norma ASTM C 778 - 2006 está compuesta básicamente por granos naturalmente redondeados de cuarzo casi puro, con un tamaño de partícula en el rango de $1.18 \mathrm{~mm}$ a $150 \mu \mathrm{m}$, y es utilizada para preparar morteros en el ensayo de cementos hidráulicos. Se empleó el agua destilada para el mezclado, para evitar el ingreso de compuestos agresivos, que cumple con la norma NMX-C-122 ONNCCE 2004. Se produjeron 5 especímenes de morteros cilíndricos de $5 \mathrm{~cm} \times 13 \mathrm{~cm}$ para cada tipo de 
cemento. Se adoptó la relación $\mathrm{a} / \mathrm{c}=0.65$ y la relación arena/mc=2.75 (Véase Tabla 2), conforme establece la ASTM 1012 - 2013. Tras el colado los especímenes permanecieron en laboratorio, durante $24 \mathrm{~h}$ a $\mathrm{T}=23^{\circ} \mathrm{C}$ para fraguar, como establece la ASTM C 171 - 2016. Además, se produjeron especímenes de morteros de $3.0 \times 8 \mathrm{~cm}$ con la misma relación a/c para los análisis microestructurales.

Tabla 2 - Materiales empleados para la producción de cilindros de morteros

\begin{tabular}{|c|c|}
\hline Material & Cantidad (g) \\
\hline Cemento & 180 \\
\hline Arena & 495 \\
\hline Agua & 117 \\
\hline
\end{tabular}

Después de $24 \mathrm{~h}$ de colado, se procedió a realizar un pretratamiento de los especímenes para la aplicación de los tratamientos superficiales.

\subsection{Preparación de especímenes y aplicación de tratamientos superficiales con NS y NF}

Para la aplicación de los tratamientos superficiales, primeramente se procedió a preparar la superficie de los especímenes. Se realizó cortes transversales a $1.5 \mathrm{~cm}$ en cada una de las extremidades de los cilindros, para evitar los efectos de frontera debido a la preparación y el colado de los especímenes. Posteriormente, se realizó una limpieza con cepillo y agua del grifo para remover el polvo que se generó en la superficie del espécimen. A continuación, se aplicó los tratamientos superficiales de los especímenes, conforme se presenta en la Tabla 3.

\subsection{Exposición al ataque físico de sulfatos}

Dado que la ASTM C1012 - 2013 abarca solamente el ataque químico de sulfatos, para evaluar el ataque físico se adoptó el procedimiento reportado por estudios previos (Nehdi et al. 2014; Zeidan et al., 2016), que tiene duración de 6 meses. El método propuesto consistió en someter cilindros de morteros de dimensiones de $5 \mathrm{~cm} \times 10 \mathrm{~cm}$ a una solución de sulfato de sodio para inducir el ataque físico, mediante ciclos de mojado y secado, que tenía duración de 4 semanas, en las siguientes condiciones: la primera condición consistió en un ambiente con temperatura de $\mathrm{T}=20^{\circ} \mathrm{C}$ y una humedad relativa de $\mathrm{HR}=82 \%$ (mojado), la segunda condición, a su vez, consistió en un ambiente con una temperatura de $\mathrm{T}=40^{\circ} \mathrm{C}$ y una humedad relativa de $\mathrm{HR}=31 \%$ (secado). Se eligió únicamente evaluar la exposición de morteros al sulfato de sodio ya que investigaciones anteriores reportaron que, respecto a la acción física, este sulfato es el más dañino a las estructuras de concreto reforzado-ECR. Por lo que, una vez que se cumplió el tiempo de curado de 28 días, los especímenes permanecieron en laboratorio a temperatura ambiente hasta alcanzar el peso constante. Posteriormente, se sometieron parcialmente ( $\pm 5 \mathrm{~mm}$ del espécimen) los especímenes a una solución al $5 \%$ de $\mathrm{Na}_{2} \mathrm{SO}_{4}$, para inducir la succión capilar de la solución. Además, se selló las cajas que contenía los especímenes, para evitar la evaporación de la solución, que a continuación fueron introducidas a una cámara ambiental modelo THERMO 3911, para promover ciclos de mojado y secado, previamente establecidos. Semanalmente, se cambió la solución de las cajas con el fin de mantener la misma concentración de la solución durante todo el tiempo de exposición. Al final de cada ciclo, que corresponde a 2 períodos de secado y dos períodos de mojado, se midió las masas y se realizó una inspección visual de los especímenes. En total, fueron realizados 6 ciclos de mojado y secado, cumpliendo los 6 meses de exposición. 
Tabla 3 - Tratamientos superficiales

\begin{tabular}{|c|l|}
\hline Nomenclatura & \multicolumn{1}{c|}{ Tipo de solución } \\
\hline $\mathbf{R F}$ & Curado estándar con agua potable \\
\hline $\mathbf{T 1}$ & $\begin{array}{l}\text { Tratamiento superficial por 72h, con una dispersión al 0.1\% de NS respecto } \\
\text { al volumen de agua desionizada (aplicado por inmersión). }\end{array}$ \\
\hline $\mathbf{T 2}$ & $\begin{array}{l}\text { Tratamiento superficial a los 28 días de curado con una solución al 0.1\% de } \\
\text { NF respecto al volumen de agua desionizada (aplicado con brocha). }\end{array}$ \\
\hline $\mathbf{T 3}$ & $\begin{array}{l}\text { Tratamiento superficial durante las primeras 72h con NS y después de 28 } \\
\text { días con NF. }\end{array}$ \\
\hline
\end{tabular}

\section{RESULTADOS}

\subsection{Caracterización fisicoquímica de las nanopartículas}

El análisis FRX, confirmó la composición del material que presentó $99.9 \%$ de $\mathrm{SiO}_{2}$. Asimismo, el difractograma de NS en el intervalo de $2 \theta=10^{\circ}$ a $60^{\circ}$ (véase Figura 1), demuestra una reflexión muy ancha ubicada en $2 \theta=22,2^{\circ}$ característica de un material base sílice, además la base del difractograma confirma la naturaleza amorfa del material. Este resultado concuerda con los difractogramas encontrados por otros autores, que produjeron NS mediante la ruta sol-gel y obtuvieron un material con características similares (Jo et al., 2007; Quing et al., 2007; CruzMoreno et al., 2017). Cabe mencionar que la funcionalización de las NS no modifica su estructura amorfa, sino cambia su energía superficial al sustituir los grupos $\mathrm{O}-\mathrm{H}$ por $\mathrm{O}-\mathrm{CH}_{3}$, así que el DRX sólo refleja el difractograma de NS. Además, mediante la técnica TEM se comprueba que se produjo NS amorfa, de tamaño nanométrico (tamaños inferiores a 100nm), con morfología irregular (véase Figura 2a y 2b), lo mismo se observó para las NF (véase Figura 2c y 2d).

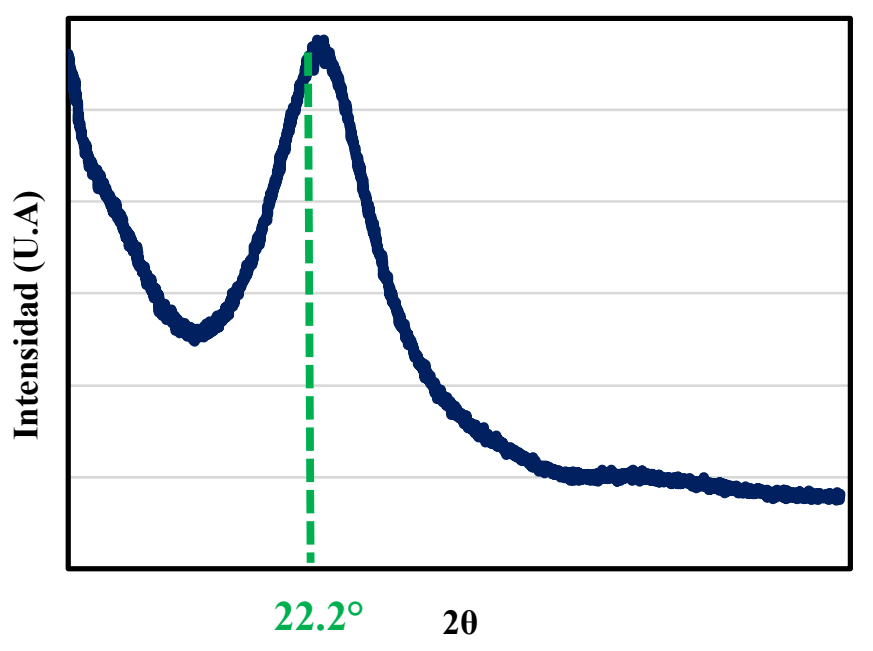

Figura 1 - Difractograma de NS

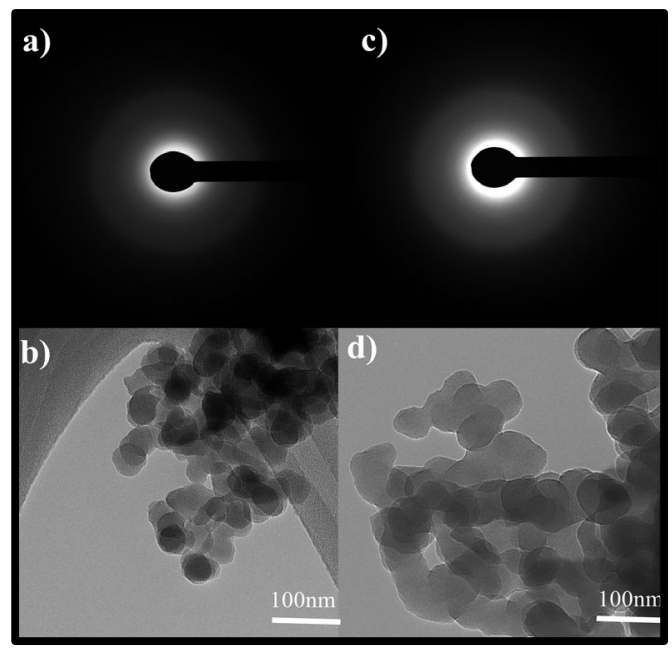

Figura 2 - a) Patrón de difracción de NS; b) Morfología de NS; c) patrón de difracción de NF; d) Morfología de NF.

Mediante el espectro de infrarrojo de la Figura 3 es posible observar las bandas de absorción presentes en el intervalo de $500 \mathrm{~cm}^{-1}$ a $4000 \mathrm{~cm}^{-1}$ características de los grupos funcionales de las NS y NF. Con los resultados de este análisis también es posible verificar la presencia de residuos 
de solventes de la síntesis. Para las NS, las bandas de absorción ubicadas en el intervalo de 1500 $\mathrm{cm}^{-1}$ a $3500 \mathrm{~cm}^{-1}$ corresponden a los enlaces $\mathrm{O}-\mathrm{H}$ y $\mathrm{C}-\mathrm{H}$, que están asociados a los reactivos empleados como solventes (Cruz-Moreno et al., 2020; Yoshino et al., 1990). En la etapa de condensación, la unión de una molécula prehidrolizada con el alcóxido resulta en la formación de enlaces Si-O-Si, cuyas bandas de absorción se ubican en $998 \mathrm{~cm}^{-1}$ y $879 \mathrm{~cm}^{-1}$, conforme indica Norazmi et al., 2018).

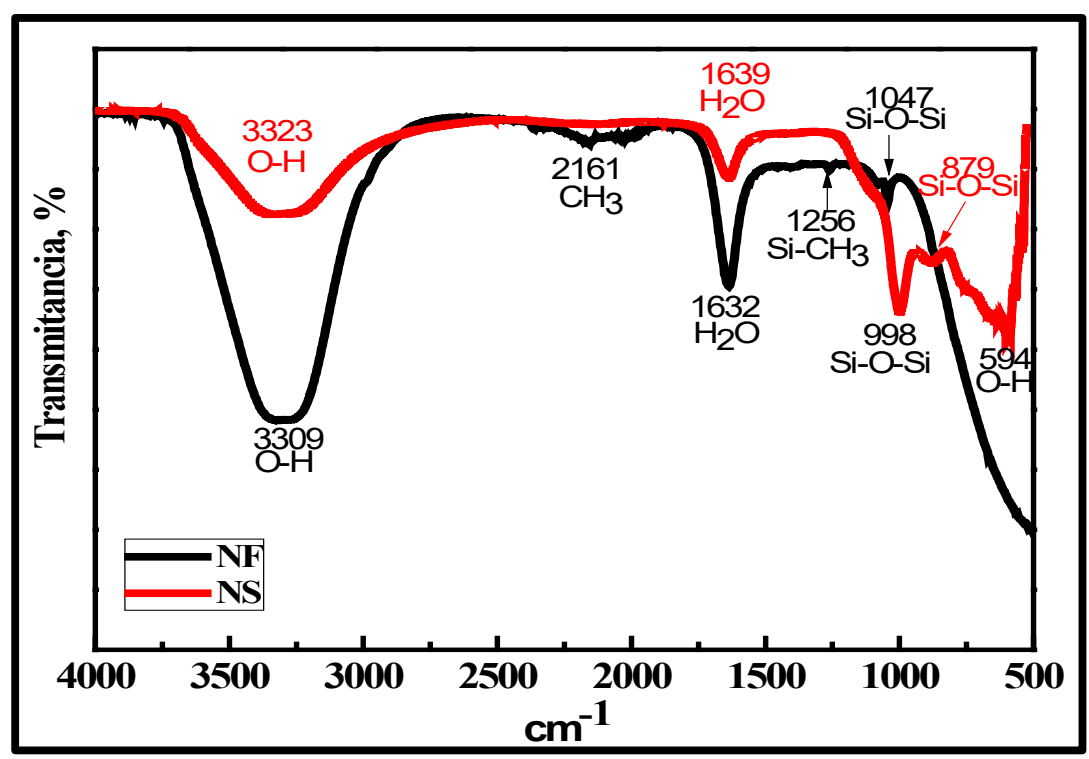

Figura 3 - Espectros obtenidos por FTIR de NS y NF

Otra banda ubicada en $594 \mathrm{~cm}^{-1}$ es atribuida a grupos alcóxido y $\mathrm{OH}$ que se quedaron en los geles (Yoshino et al., 1990; Decottiginies et al., 1978). Aún en la Figura 3, se observa el efecto generado por la funcionalización de NS, con la aparición de los picos ubicados en las longitudes de $2161 \mathrm{~cm}^{-}$ ${ }_{1}^{1}$ correspondientes a las vibraciones de los radicales $\mathrm{CH}_{3}$, y a las vibraciones pertenecientes a los radicales $\mathrm{Si}-\mathrm{CH}_{3}$, ubicados en $1256 \mathrm{~cm}^{-1}$. Estos grupos funcionales son responsables por la disminución de la energía superficial o de contacto en el concreto, brindándole el efecto hidrofóbico. Por lo tanto, en este análisis se observó que el material formado corresponde a las NS y NF, dado que el espectro FTIR confirma la presencia de grupos funcionales de este material.

\subsection{Ataque físico de sulfatos}

\subsubsection{Inspección visual}

Se conoce que el ataque físico de sulfatos se ubica en la zona de evaporación del espécimen (región A), mientras que en la porción inmersa en la solución (región B) podrá o no ocurrir el ataque químico (Nehdi et al., 2014) (Véase Figura 4).

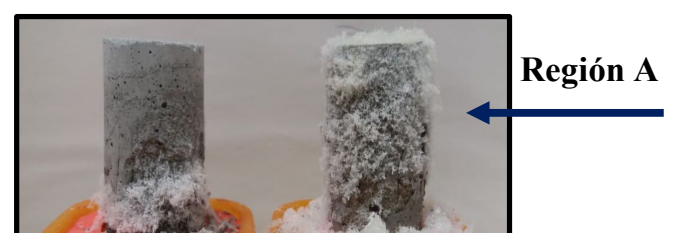

XVI Congreso Latinoamericano de Patología de la Construcción 


\section{Figura 4 - Exposición al ataque físico de sulfatos}

El mecanismo del ataque de sulfatos empieza mediante la eflorescencia de cristales de sulfato de sodio en la superficie del mortero, siendo más evidente en el período de secado $\left(\mathrm{T}=40^{\circ} \mathrm{C}\right.$ y $\mathrm{HR}=31 \%$ ). Consecuentemente, se observa el fenómeno conocido como "scaling" que es la perdida de material superficial, debido a la presión ejercida por el crecimiento continuo de cristales de sulfato en los poros superficiales del mortero (Haynes, 2011).

Según Bassuoni (2015) esta presión de cristalización depende del tamaño de los poros, y los poros más afectados son los microporos. Conforme reporta (Haynes, 2011) la cristalización de estas sales en los poros ocurre cuando la tasa de evaporación de la superficie excede la tasa de absorción por capilaridad, debido a los cambios de temperatura y humedad relativa. Por esta razón, se ha observado una reducción en el nivel de la solución durante el tiempo de exposición de los especímenes.

También cabe mencionar que el régimen de exposición seleccionado $\left(\mathrm{T}=20^{\circ}\right.$ y $\mathrm{HR}=82 \%$; $\mathrm{T}=40^{\circ} \mathrm{C}$ y $\mathrm{HR}=31 \%$ ) coincide con el ciclo entre la fase thenardita y mirabilita del sulfato de sodio (Bassuoni, 2015). Por lo tanto, los primeros indicios de deterioro se presentaron en el $3^{\circ}$ mes de exposición, como demuestra la Figura 5, empezando en la región de evaporación, conforme la literatura. Al final de la exposición, los especímenes presentaron el "scaling" de la superficie en nivel avanzado, sobre todo para el espécimen RF del cemento CPO que llego a fracturarse. Por otro lado, en los morteros producidos con cemento RS, el espécimen tratado con el tratamiento T3 presentó el menor grado de deterioro, como demuestra la Figura 5.

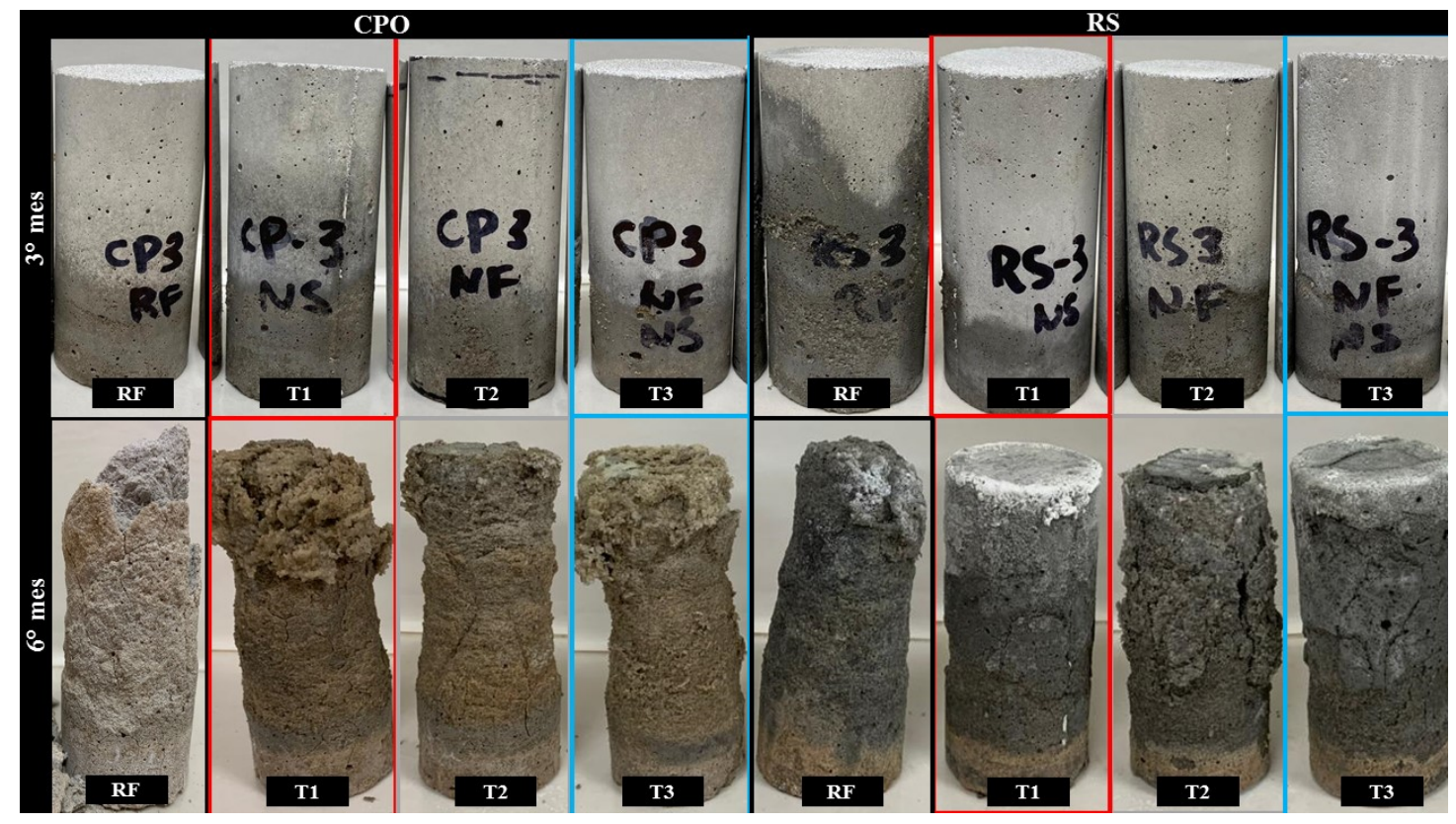

Según Ne Figura 5 - Morteros de RS y CPO sometidos al ataque físico de sulfatos. ıque físico de sulfato, ya que una ae ias caracterısticas de este ataque es eı "scalıng ae ia superiıcie del material cementante, por lo que en esta investigación se midió periódicamente la variación de masa de morteros de CPO y RS parcialmente inmersos en una solución de sulfato de sodio. Según los 
autores, en la etapa inicial de exposición los morteros absorben la solución mediante succión capilar y con el avance de la exposición, la solución migra hacia la superficie del material provocando el deterioro del material y, por ende, la pérdida de masa.

\subsubsection{Variación de masa}

La Figura 6 demuestra que hasta tres meses de exposición se observó un incremento de masa en todos los tipos de tratamiento y en el mortero de referencia, sin embargo, el mortero RF presentó un incremento más acelerado, respecto a especímenes tratados. El menor incremento de masa del mortero tratado con el tratamiento $\mathrm{T} 1$ posiblemente se debe al bloqueo de poros provocado por las NS; por otro lado, para los morteros de los tratamientos T2 y T3 se debe a la propiedad hidrofóbica de las NF, que disminuye la absorción de la solución por repeler el agua en edades tempranas de exposición (Cruz-Moreno et al., 2020). Sin embargo, con el aumento del tiempo de exposición se observó que los morteros del tratamiento T2 y T3 tuvieron una ganancia de masa comparable a los demás morteros. Posiblemente, esto ha ocurrido debido a que el material ha perdido o reducido su propiedad hidrofóbica debido al contacto constante con el agua, favoreciendo el ingreso de la solución salina hacia el interior de la matriz. La propiedad hidrofóbica se pierde debido a la conversión de los grupos funcionales $\mathrm{Si}-\mathrm{O}-\mathrm{CH}_{3}$ en $\mathrm{Si}-\mathrm{OH}$.

La Figura 6 también demuestra que entre el tercero y el quinto mes de exposición, todos los morteros presentaron pérdida de masa, siendo el mortero RF el que presentó la mayor tasa de variación (alrededor de $8 \%$ ), seguido del tratamiento T2 que presentó una tasa de variación de $6 \%$. Por otro lado, los tratamientos T1 y T3 presentaron las menores pérdidas de masa, infiriendo que estos tratamientos son más favorables a la mitigación del ataque físico de sulfato. Este resultado es congruente con el resultado reportado por Zeidan et al. (2016), sin embargo Nehdi et al. (2014) ha reportado que para concretos producidos con una relación $\mathrm{a} / \mathrm{c}=0.60$ la pérdida de masa fue del orden de $3 \%$ para la referencia del cemento CPO.

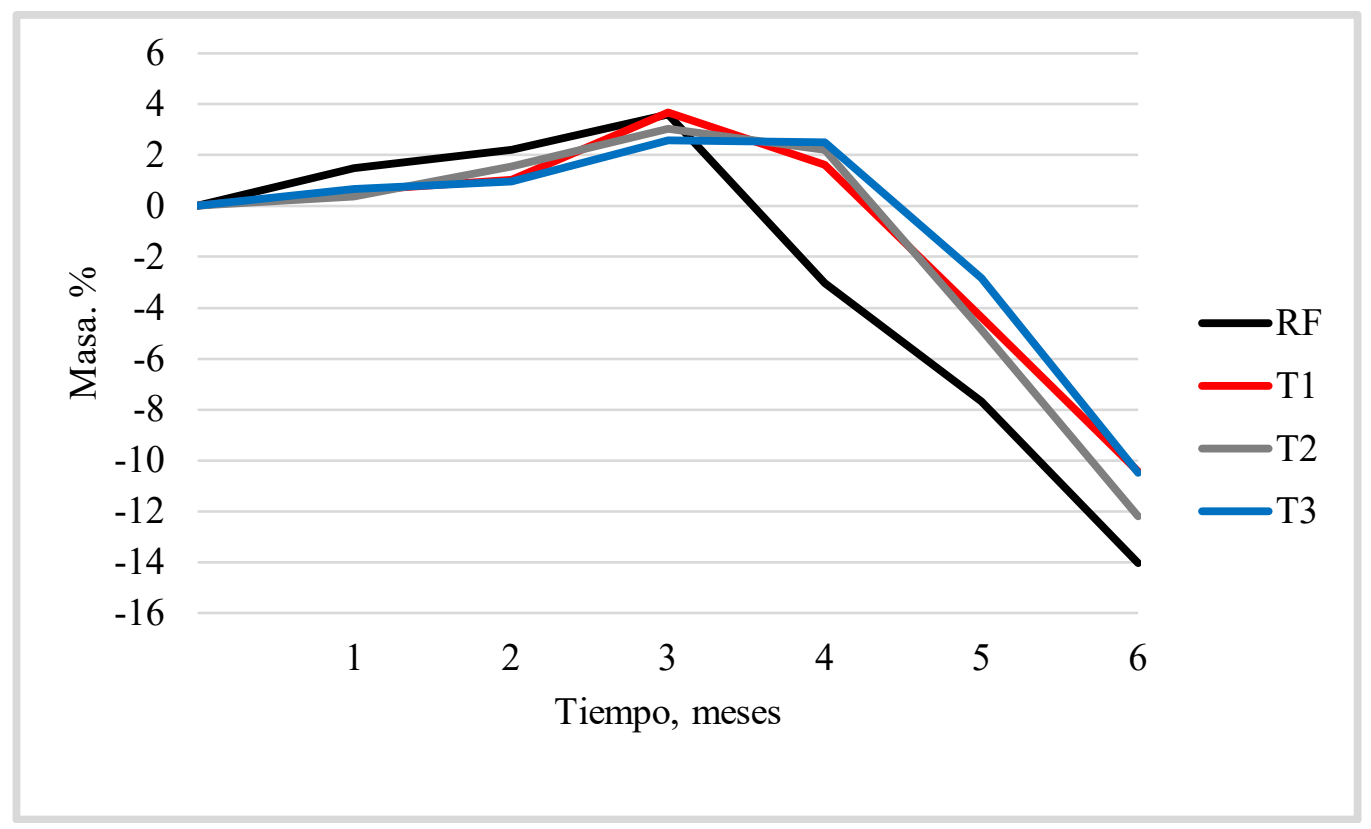

Figura 6 - Variación de masa de los morteros de CPO.

A su vez, como se observa en la Figura 7, los morteros de RS ganaron masa desde el primer mes de exposición, siendo el tratamiento T3 el que presentó la menor ganancia de masa. En el segundo 
mes de exposición, se observa que a excepción del mortero T3, todos los demás empezaron a perder masa, siendo el mortero RF el que presentó la mayor pérdida. Después de 6 meses de exposición, se observó que los morteros tratados con los tratamientos que contienen NS (T1 y T3) presentaron la menor pérdida de masa respecto a la referencia que presentó una pérdida alrededor del 18\%.

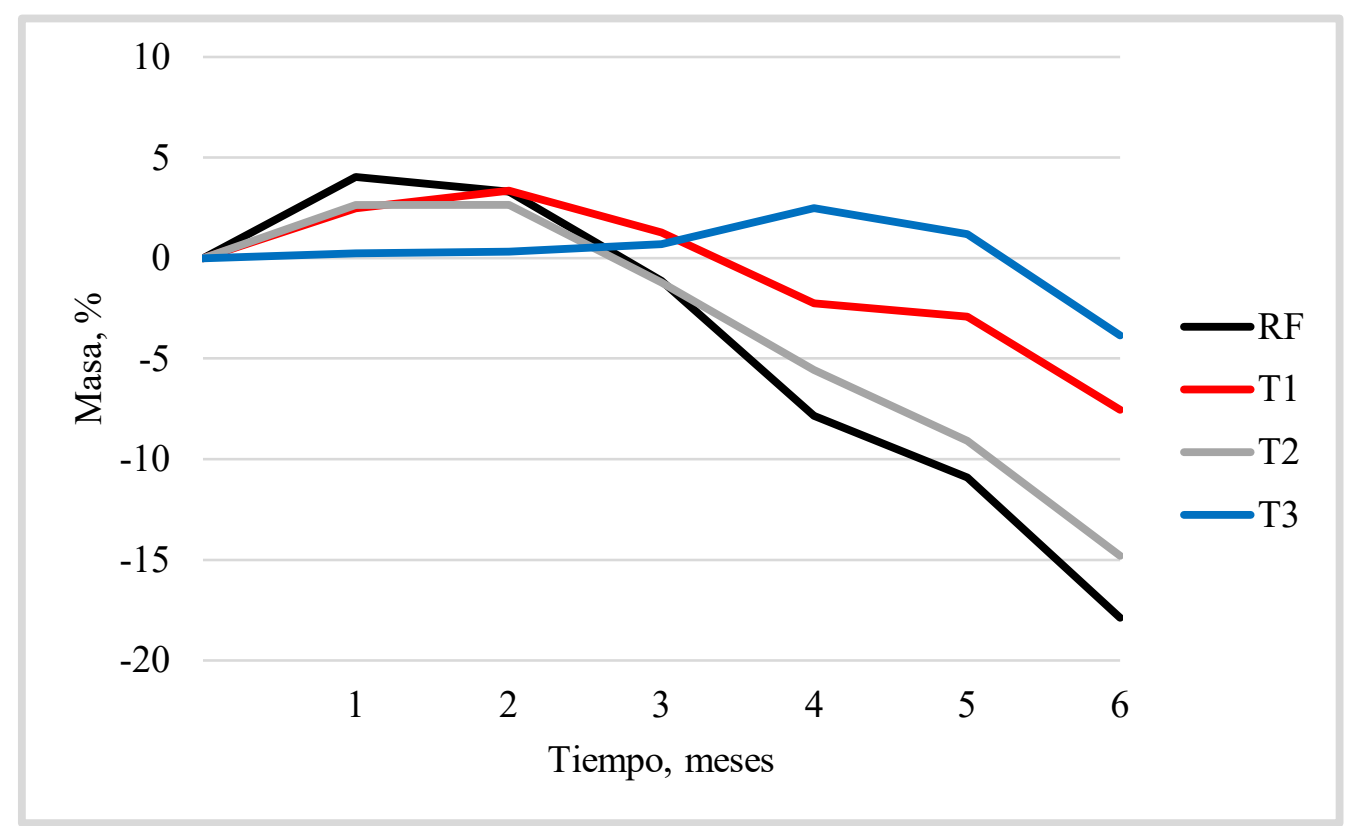

Figura 7 - Variación de masa de los morteros de RS.

De una manera general, los valores de pérdida de masa están de acuerdo con la apariencia visual del daño en la superficie de los especímenes, es decir los morteros que presentaron la mayor pérdida masa también presentaron el mayor grado de deterioro, esto confirma lo que ha sido reportado por Zeidan et al., (2016). Finalmente, con lo que se observó en los análisis de variación de masa es posible predecir que, para las condiciones seleccionadas, el tratamiento T1 y T3 se presentan más favorables para la mitigación del ataque físico de sulfatos.

\subsection{3 Ángulo de contacto}

En la medición del ángulo de contacto (véase Figura 8) se observó que para los morteros de CPO, en la Región A, los ángulos disminuyeron, respecto a la medición inicial, pero aún se mantienen en la zona hidrofóbica $\left(\theta>90^{\circ}\right)$ hasta los dos meses de exposición. En la región B que está inmersa en la solución sulfática, estos ángulos se redujeron para la zona hidrofílica $\left(10^{\circ}<\theta<90^{\circ}\right)$. Asimismo, para los morteros de RS, los ángulos de contacto de la región $\mathrm{A}$, se mantienen en la zona hidrofóbica (véase Figura 9) hasta los 2 meses de exposición, mientras la región $\mathrm{B}$, que está inmersa en la solución sulfática, presentó ángulos de contacto inferiores a $90^{\circ}$. La disminución de los ángulos de contacto en la región B para la zona hidrofílica, indica que el contacto constante con la solución puede provocar la pérdida de hidrofobicidad favoreciendo el paso de solución salina hacia el interior del espécimen. Por otro lado, aunque la región B haya perdido la hidrofobicidad en el primer mes de exposición, se observó en el análisis de variación de masa que los morteros de los tratamientos T1 y T3 ganaron menos masa respecto a la referencia, indicando que estos tratamientos han afectado el ingreso de solución. Cabe mencionar que, a partir del tercer mes de exposición, cuando empezó a presentarse el deterioro provocado por el ataque físico de sulfatos, ya no se ha logrado medir los ángulos de contacto en la región B de los especímenes evaluados. 


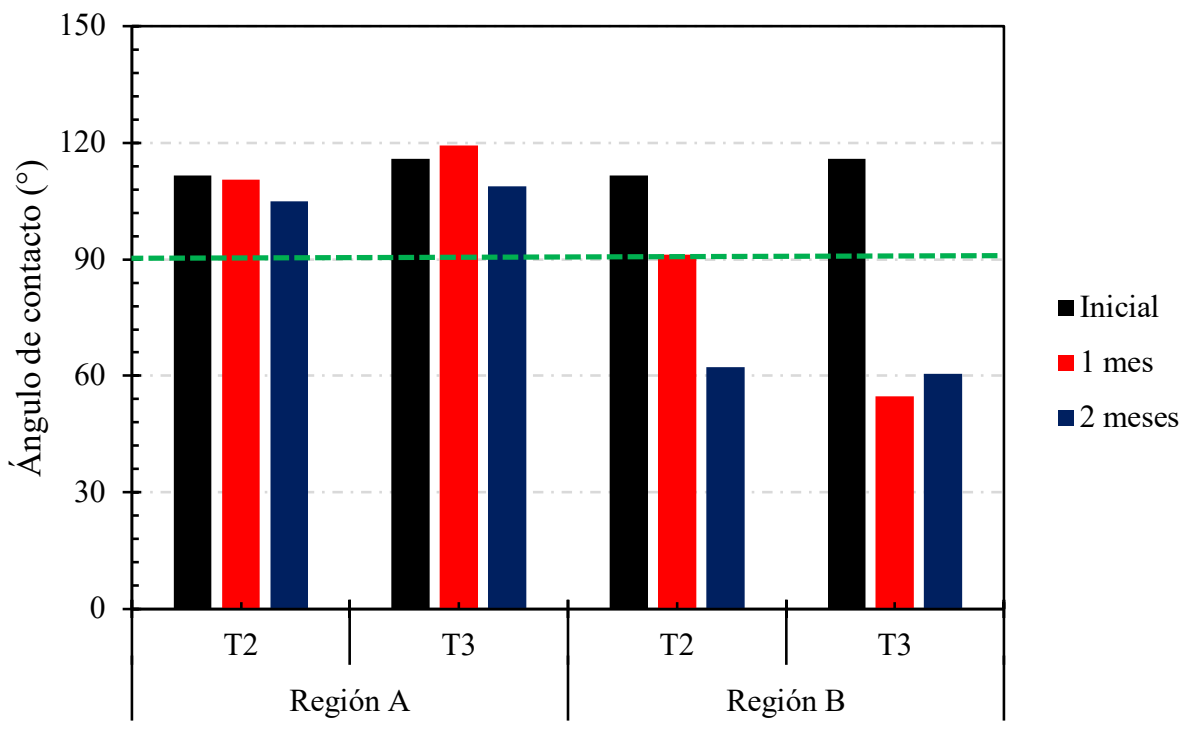

Figura 8 - Ángulo de contacto - CPO

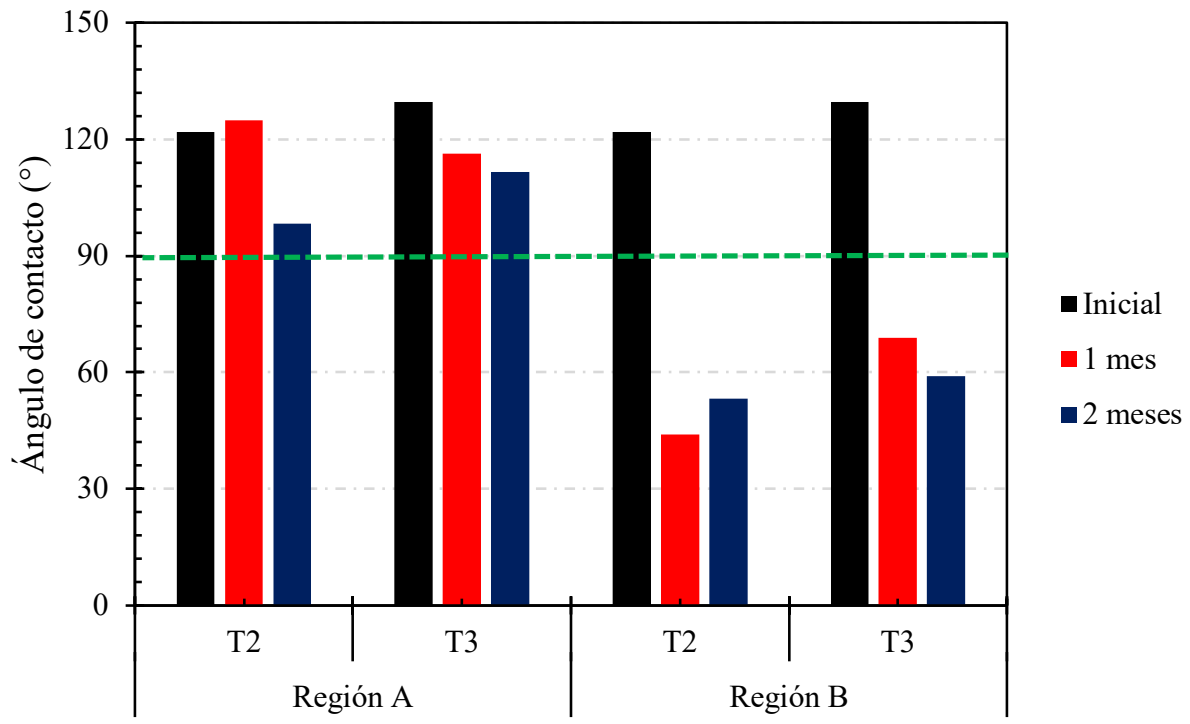

Figura 9 - Ángulo de contacto - RS

\subsubsection{Sortividad}

Según Bassuoni (2015), la absorción total de agua puede usarse como un indicador aproximado de la resistencia del concreto al ataque físico de sulfatos, por lo que se midió la sortividad de especímenes de morteros producidos con cemento CPO y RS. Cabe mencionar que la absorción representa la porosidad efectiva o accesible al agua y por ende se relaciona al ingreso de los agentes agresivos ambientales. La Tabla 4 demuestra que la tasa de absorción inicial y secundaria no presentó una reducción significativa al comparar las muestras tratadas con respecto a la referencia, para los dos tipos de cemento. Sin embargo, fue observado a las $6 \mathrm{~h}$ de exposición al agua, en donde tiene lugar la absorción inicial, que los morteros tratados con los tratamientos T2 y T3 absorbieron menos agua que los demás, demostrando que hubo un retraso en el ingreso de agua hacia el interior 
del espécimen. Posiblemente esto se debió al efecto hidrofóbico que brindan las NF a los morteros de tratamientos T2 y T3. Por otro lado, a los 8 días de análisis, en donde tiene lugar la absorción secundaria, fue observado que los especímenes tratados con los tratamientos T1 y T3 absorbieron menos agua, infiriendo que las NS presentes en ambos tratamientos han contribuido para densificar la matriz de cemento y reducir las interconexiones de los poros capilares. Cabe mencionar que, al comparar las gráficas de variación de masa del cemento CPO con los resultados de absorción, se observa que el incremento tardío de masa de los morteros tratados con los tratamientos T1, T2 y $\mathrm{T} 3$, se debe posiblemente a la menor absorción de agua total que estos morteros presentaron, sobre todo en las primeras edades, como comprobó el análisis de sortividad.

Tabla 4 - Sortividad

\begin{tabular}{|c|c|c|c|c|c|}
\hline \multicolumn{7}{|c|}{ Tasa de absorción, $\times \mathbf{1 0}^{-\mathbf{4}} \mathbf{~ m m} / \mathbf{s}^{\mathbf{1 / 2}}$} \\
\hline Cemento & Absorción & $\mathrm{RF}$ & $\mathrm{T} 1$ & $\mathrm{~T} 2$ & $\mathrm{~T} 3$ \\
\hline \multirow{2}{*}{ CPO } & Inicial & 177.1 & 154.0 & 164.5 & 149.07 \\
\cline { 2 - 6 } & Secundaria & 74.5 & 68.9 & 75.7 & 67.10 \\
\hline \multirow{2}{*}{ RS } & Inicial & 187.6 & 178.5 & 161.4 & 138.30 \\
\cline { 2 - 6 } & Secundaria & 87.4 & 76.3 & 85.3 & 75.10 \\
\hline
\end{tabular}

De manera general, se demostró que los tratamientos utilizados fueron favorables a la mitigación del ataque físico de sulfatos. Para el caso del tratamiento T1, que contiene NS, posiblemente esto se debió a la densificación de la capa superficial del concreto, que juega un papel fundamental en lo que es la succión capilar de agua, que es el primer paso del ataque físico de sulfatos en donde la solución penetra al mortero. Asimismo, para el caso del tratamiento T2, que contiene NF, se observó que el efecto del ataque físico fue menor, comparado con la referencia. Pero, en este caso ya que no hay interacción química del tratamiento con la superficie del mortero, desarrollando una reacción puzolánica, posiblemente al impedir el ingreso de agua en las primeras edades mediante el efecto hidrofóbico, conllevó a la reducción de los efectos del ataque físico de sulfatos, presentando la segunda mayor tasa de variación de masa. En contrapartida, el tratamiento T3, que contiene NS y NF, por brindar doble protección al material, la primera por bloquear los poros superficiales y la segunda por brindar el efecto hidrofóbico, se presentó más favorable a la mitigación de la acción física del sulfato de sodio.

\section{CONCLUSIONES}

Se puede concluir que las nanopartículas que se obtuvieron mediante la síntesis sol-gel presentan características deseadas. Por lo anterior se concluye que, mediante el análisis de sortividad del material se observó que las matrices tratadas con nanopartículas de sílice, sea NS o NF, absorbieron una menor cantidad de agua, indicando que los tratamientos utilizados han demostrado ser factibles a disminuir el ingreso de agua hacia el interior de la matriz cementante. En la medición de ángulo de contacto se observó que la porción del espécimen en contacto con la solución salina, presentaron una reducción del ángulo de contacto en el primer mes de exposición, indicando que el contacto directo con la humedad puede afectar el tratamiento superficial con NF. Asimismo, se observó en el análisis de pérdida de masa que los especímenes tratados con NS y NF presentaron una menor pérdida de masa al final de la exposición al ataque físico de sulfatos. Esto indica que el tratamiento brindó una protección al mortero ante a la acción física de sulfato de sodio. 


\section{AGRADECIMIENTOS}

Los autores expresan su agradecimiento al fondo SEP-CONACyT por el financiamiento otorgado al proyecto CB-2016/285453. D. L. Silva agradece a CONACyT por la beca ( $\mathrm{n}^{\circ}$ 932159) otorgada para la realización de sus estudios doctorales.

\section{REFERENCIAS}

ASTM International. (2006). ASTM C778-06 Standard Specification for Standard Sand; https://doi.org/10.1520/C0778-06.

ASTM International. (2007). ASTM C 150-07 Standard specification of portland cement; https://doi.org/10.1520/C150-07.

ASTM International. (2013). ASTM - C 1012 Standard Test Method for Length Change of Hydraulic Cement Mortar Exposed to Sulfate Solution. https://doi.org/10.1520/C1012.

ASTM International. (2016). ASTM C 171 - 16 Standard specification for sheet materials for curing concrete. https://doi.org/10.1520/C171-16.

Atahan H. N., Dikme, D. (2011). Use of mineral admixtures for enhanced resistance against sulfate attack, Constr. Build. Mater. 25 (8). 3450-3457.

Cruz-Moreno, D., Fajardo, G., Flores-Vivián, I., Cruz-López, A., Valdez, P. (2017), Tratamiento superficial con nanopartículas base silicio inducido durante el curado: Efecto en la durabilidad de materiales base cemento portland, Revista ALCONPAT, 7(3):274-285, http://dx.doi.org/10.21041/ra.v7i3.239.

Cruz-Moreno, D., Fajardo, G., Flores-Vivián, I., Orozco-Cruz, R.; Ramos-Rivera, C.. (2020). Multifunctional surfaces of portland cement-based materials developed with functionalized siliconbased nanoparticles. Applied Surface Science. 531. https://doi.org/10.1016/j.apsusc.2020.147355. Decottignies, M.; Phalippou, J., Zarzycky, J. (1978). Synthesis of glassed by hot-pressing of gels. J. of Materials Science 13, 2605-2618.

Fajardo, G., Cruz-López, A., Cruz-Moreno, D., Valdez, P., Torres, G., Zanella, R. (2015), Innovative application of silicon nanoparticles (SN): Improvement of the barrier effect in hardened Portland cement based materials. Construction and Building Materials, 76, 158-167.

Gómez-Zamorano, C., Castillo-Linton, E. (2016). Modificación de las propiedades de matrices cementantes mediante la adición de partículas de nanosílice. Revista ALCONPAT. Volumen 6(2): 101-115.

Haynes H., Bassuoni MT (2011). Physical salt attack on concrete. Concr. 33: 38-42.

Haynes H., O’Neill R, Neff M. (2008) Salt weathering distress on concrete exposed to sodium sulfate environment. ACI Mater. 105:35-43.

Jalal M., Mansouri E, Sharifipour M, Pouladkhan AR (2012). Mechanical, rheological, durability and microstructural properties of high performance self-compacting concrete containing $\mathrm{SiO}_{2}$ micro and nanoparticles. Mater Des 2012;34: 389-400.

Jo B. W.; Kim C. H.; Tae G. H.; Park J. B. (2007). Characteristics of cement mortar with nanoSiO 2 particles". Constr. Build. Mater., 21:1352-5.

Li, L. G.; Zhu, J.; Huang, Z. H.; Kwan, A. K. H.; Li, L. J. (2017). Combined effects of microsilica and nano-silica on durability of mortar, Constr. Build. Mater. 157, 337-347.

Bassuoni, M., Rahman M., (2015), Response of concrete to accelerated physical salt attack exposure, Cem. Concr. Res. http://dx.doi.org/10.1016/j.cemconres.

Nehdi, M. L.; Suleiman, A.R. (2014) Soliman. Investigation of concrete exposed to dual sulfate attack. Cement and Concrete Research 64, 42-53. 
NMX-AA-122-ONNCCE- 2006, Potabilización del agua para uso y consumo humano - sulfato de aluminio - especificaciones y métodos de prueba, Organismo Nacional de Normalización y Certificación de la Construcción y Edificación.

NMX-C-414-ONNCCE- 2004, Industria de la Construcción - Cementos Hidráulicos Especificaciones y Métodos de Prueba, Organismo Nacional de Normalización y Certificación de la Construcción y Edificación, S.C., Febrero 2005.

Ribeiro, D. V., Sales, A., Sousa, C. A. C., Cunha, M. P. T., Lourenço, M. Z., Helene, P. (2014). Corrosão em estruturas de concreto armado: Teoria, controle e métodos de análise. Ed. Elsevier, Rio de Janeiro.

Scherer, G. W., (2004), Stress from crystallization of salt, Cement and Concrete Research, Vol. 34, No. 9, pp.1613-1624.

Thaulow, N., Sahu, S., (2004), Mechanism of concrete deterioration due to salt crystallization, Materials Characterization, Vol. 53, No. 2-4, pp. 123-127.

Tobon, J. Payá, O. J. (2015), Restrepo, Study of durability of Portland cement mortars blended with silica nanoparticles, Constr. Build. Mater. 80, 92- 97.

Qing Y., Zenan Z., Deyu K. (2007), Influence of nano-SiO ${ }_{2}$ addition on properties of hardened cement paste as compared with silica fume. Construction and Building Materials. 21: 539-45. 12. KL.

Yoshida, N., Matsunami, Y., Nagayama, M., and Sakai, E., (2010), Salt weathering in residential concrete foundation exposed to sulfate-bearing ground, Journal of Advanced Concrete Technology, Vol. 8, No. 2, pp. 121-134.

Yoshino, H.; Kamiya, K. Y Nasu, H. (1990), IR study on the estrucral evolution of sol-gel derivaded $\mathrm{SiO}_{2}$ gels in the early stage of conversion to glasses. J. Non-Cryst. Solids 126, 68-78.

Zeidan, M.; Mohamed T. Bassuoni; Aly Said (2016). Physical salt attack on concrete incorporating nano-silica. Jour. of Sust. Cem.-Based Mater.. http://dx.doi.org/10.1080/21650373.2016.1218802 